2016-03-15

Cage-induced stereotypic behaviour in

laboratory mice covaries with nucleus accumbens FosB/FosB expression.

\title{
Phillips, D
}

http://hdl.handle.net/10026.1/10412

10.1016/j.bbr.2015.12.035

Behav Brain Res

All content in PEARL is protected by copyright law. Author manuscripts are made available in accordance with publisher policies. Please cite only the published version using the details provided on the item record or document. In the absence of an open licence (e.g. Creative Commons), permissions for further reuse of content should be sought from the publisher or author. 
2 Cage-induced stereotypic behaviour in laboratory mice covaries with nucleus accumbens FosB/ $\Delta$ FosB expression

6 Danielle Phillips ${ }^{1}$, Elena Choleris ${ }^{1}$, Kelsy S. J. Ervin ${ }^{1}$, Carole Fureix ${ }^{2}$, Laura Harper ${ }^{3}$, Kathryn Reynolds ${ }^{4}$, Lee $\mathrm{Niel}^{5}$, and Georgia J. Mason ${ }^{3 *}$

$12{ }^{1}$ Department of Psychology and Neuroscience Program, University of Guelph, Ontario

132 Department of Animal Husbandry, University of Bristol, Langford, UK

143 Department of Animal Biosciences, University of Guelph, Ontario

154 Department of Molecular \& Cellular Biology, University of Guelph, Ontario

165 Department of Population Medicine, University of Guelph, Ontario

17

18

19

20

$21 *$ Contact for correspondence: gmason@uoguelph.ca 


\section{Abstract}

26 Stereotypic behaviour (SB) occurs in certain human disorders (e.g. autism), and animals

27 treated with stimulants or raised in impoverished conditions, including laboratory mice in

28 standard cages. Dysfunctional cortico-basal ganglia pathways have been implicated in

29 these examples, but for cage-induced forms of SB, the relative roles of ventral versus

30 dorsal striatum have not been fully ascertained. Here, we used immunohistochemical

31 staining of FosB and $\Delta$ FosB to assess long-term activation within the nucleus accumbens

32 and caudate-putamen of C57BL/6 mice. Housed in typical laboratory cages, these mice

33 spontaneously developed different degrees of route-tracing, bar-mouthing and other

34 forms of SB (spending $0 \%$ to over $50 \%$ of their active time budgets in this behaviour).

35 The most highly stereotypic mice showed the most elevated FosB/ $\Delta$ FosB activity in the

36 nucleus accumbens. No such patterns occurred in the caudate-putamen. The cage-induced

37 SB common in standard-housed mice thus involves elevated activity within the ventral

38 striatum, suggesting an aetiology closer to compulsive gambling, eating and drug-seeking

39 than to classic amphetamine stereotypies and other behaviours induced by motor loop

40 over-activation.

43 Key words: Stereotypic behaviour; stereotypy; abnormal repetitive behaviour; nucleus

44 accumbens; caudate-putamen; striatum; transcription factor; $\Delta$ FosB 
Stereotypic behaviour (SB) is common in some human neurodevelopmental disorders

49 (e.g. autism), where it seems to reflect dysfunctional basal ganglia circuitry [1, 2]. It can

50 also be experimentally induced in animals by manipulating these circuits [e.g. via

51 stimulants 2, 3]. SB is common, too, in farm, zoo and laboratory animals raised and

52 housed in impoverished enclosures [1, 2, 4]. Here, SB often covaries with generalized

53 signs of impaired behavioural control [1, 4, 5], again suggesting cortico-striatal

54 dysfunction. Furthermore, the types of enclosure that promote SB induce diverse

55 structural and biochemical changes within the basal ganglia [2, 6, 7]. However, more

56 direct evidence for basal ganglia involvement, based on neurological differences that

57 correlate with SB at the individual level, is rare, and comes from just two species: the

58 deer mouse (Peromyscus maniculatus) and horse (Equus caballus). The deer mouse

59 studies found that animals spontaneously developing high levels of SB, compared to

60 those displaying little or none, have reduced cytochrome oxidase (CO) activity in the

61 ventromedial caudate-putamen $(\mathrm{CPu})$, reduced $\mathrm{CO}$ activity in subthalamic nuclei, and

62 altered $\mathrm{CPu}$ dynorphin/encephalin ratios consistent with reduced inhibition in cortico-

63 striatal 'motor loops' [2, 6, 7]. Complementary studies highlighted changes in the frontal

64 cortex, including deficient glutathione systems [8]. Equine research, focussing on

65 dopamine receptor densities, has instead revealed elevated D1 and D2 densities within the

66 nucleus accumbens (NAc) of highly stereotypic horses [1], alongside unexpectedly lower

67 D1 densities in the dorso-medial striatum.

68 Over 30 million mice of the Mus genus are used annually in research worldwide,

69 and in standard housing many display SB [9]. Despite this, the neurological bases of

70 laboratory mouse SB are essentially unknown. In one study, the tendencies of two strains,

71 C57BL/6 (henceforth 'C57') and DBA, to develop SB were contrasted, and strain-typical

72 predispositions to high levels suggested to reflect strain differences in the up-regulation

73 of NAc dopamine receptors [3, 10]. Consistently, a second experiment drew parallels

74 between the high SB of C58BL/6 mice compared to other strains, and its enhanced

75 locomotor responses to amphetamine [11]. Neither study, however, looked at within-

76 strain individual differences or obtained strong evidence of causality. A third study

77 revealed distinct individual behavioural correlates of SB in C57s: elevated response

78 repetition in two-choice 'gambling' tasks in the most stereotypic mice [5] (something 
other behavioural studies failed to replicate in another strain, CD-1 [12,13]). This effect

80 in the stereotypic C57s was interpreted as reflecting dorsal striatal dysfunction [5]. This

81 summarises what little is known about cage-induced SB in Mus. We therefore compared

82 spontaneously high and low SB C57 mice, using immunohistochemistry to assay FosB

83 and $\Delta$ FosB. Fos family proteins are transcription factors that regulate gene expression,

$84 \Delta$ FosB being a highly stable FosB variant that accumulates over time with repeated

85 stimulation, and that mediates long-term neuronal plasticity [e.g. 14, 15, 16]. $\Delta$ FosB thus

86 reflects long-term neuronal activation, in a manner perhaps likely to covary with CO [7].

87 We investigated whether, within the basal ganglia, the caudate-putamen and/or the

88 nucleus accumbens show evidence of sustained activation in highly stereotypic mice.

89 Protocols were approved by the University of Guelph’s Animal Care Committee.

9030 C57 females (from Charles River, Quebec) were housed from 4 weeks of age in mixed

91 strain triplets (for the purposes of another study: two C57s plus one DBA/2; see [17] for

92 validation), in 15 standard woodchip-bedded laboratory cages (12cm H X 27L x 16W),

93 each provided with Shepherd Enviro-dri ${ }^{\odot}$ nesting material and a UDEL polysulfone

94 plastic mouse house shelter, plus ad libitum food (Harlan ${ }^{\circledR}$ Teklad Global Diet) and

95 water. One C57 per cage was ear-notched for identification. Cages were maintained at

$9621^{\circ} \mathrm{C}$, on a 12:12 reversed dark/light cycle (lights off at 1000h). After five months,

97 behavioural observations were conducted. Mice were live scan-sampled in situ, using red

98 room lights/headlamps, to assess activity budgets. Scans were taken every 20 minutes, for

99 four hours per session (1130-1530h or 1730-2130h), repeated over four days (thus eight

100 sessions): a method based on [17]. Scans were split between two experimenters (LH and

101 KR; inter-observer reliability: >95\%). Table 1 provides the ethogram (modified from

102 [18]). SB was calculated as a percentage of both overall activity and observations.

103 However, these covaried tightly $\left(F_{1,15.2}=914.38, P<0.0001, R^{2}>0.99\right)$ and gave near

104 identical results, so only results for the former are presented.

105 Subjects were killed by cervical dislocation when 7 months old. Brains were

106 extracted immediately and drop-fixed in cold 4\% paraformaldehyde, PFA (cf. e.g. [19]),

107 the PFA being refreshed twice within the first $24 \mathrm{~h}$ to aid fixation. After c. 4 weeks in

108 PFA (stored at 4 degrees), brains were then transferred into cryoprotective 30\% sucrose

109 in phosphate buffered saline (PBS) for 48 hours, and tissue was then sliced using a Leica 
110 Cryostat into $30 \mu \mathrm{m}$ coronal sections collected in series, mounted onto gelled slides, and 111 stored at $-80^{\circ} \mathrm{C}$ until staining.

112 In six of the cages, both C57s were clearly stereotypic (spending 3.5-55.5\% of

113 their active time budget in SB). In the remaining nine cages, they performed negligible

114 SB (0-1.5\% of the active time budget). Data from cagemates are not statistically

115 independent [20], and correspondingly, the SB levels of the two C57s per cage tightly

116 covaried $\left(\mathrm{F}_{1,13}=111.89, \mathrm{P}<0.0001\right)$. The brain of one mouse was therefore chosen from

117 each of the six stereotypic cages, and one subject randomly chosen from five of the others

118 (by GM, to ensure the researcher performing staining and quantification [DP] was blind).

119 One slide was chosen per mouse, from which two adjacent sections were selected (using

120 landmarks [cf. 14, 21] ensuring staining of both caudate-putamen and nucleus

121 accumbens).

122 To develop the FosB/ $\triangle$ FosB immunohistochemical procedure (the antibody used

123 [Sc-48, Santa Cruz Biotechnology, CA] was anti- $\Delta$ FosB but cross-reacted with FosB),

124 protocols were obtained from authors of relevant studies [14, 22, 23, 24]. These varied, so

125 were combined and optimized via pilots on spare tissue. In the final protocol, all

126 conducted at room temperature, slides were post-fixed in 4\% PFA for 10 minutes, rinsed

127 in PBS, then exposed to $\mathrm{H}_{2} \mathrm{O}_{2}$ (1\% in PBS) for 10 minutes. The samples were blocked

128 (PBS, 1.5\% Triton X and 3\% goat serum) for 1 hour, incubated in primary antibody

129 (diluted 1:500 in PBS with 0.3\% Triton X and 0.03\% sodium azide) for 22 hours, then

130 incubated in biotinylated goat anti-rabbit secondary antibody (1:200, Vector Laboratories,

131 Burlingame, CA) for 2 hours. The amplification step used an avidin/biotin peroxidase

132 complex (Vectastain ABC Kit, Vector Laboratories) for 90 minutes. Immunoreactivity

133 was revealed by incubating slides for 12 minutes in $0.06 \% \mathrm{DAB}$ and $0.1 \% \mathrm{H}_{2} \mathrm{O}_{2}$, diluted

134 in PBS. Sections then were rinsed in PBS, dehydrated in increasing concentrations of

135 ethanol (50\% for 1 minute, 70\% for 1 minute, 95\% for 3 minutes, 100\% for 3 minutes),

136 dipped in xylene for 2 hours, then coverslipped using DPX Mountant (Fisher Scientific).

137 Additional control slides underwent each step except the primary antibody, to ensure

138 background staining was absent.

139 Images were taken using the x10 objective of a Leica DMR HC Brightfield

140 microscope, and a sample of 200x200 pixels selected from each of five regions: the NAc, 
141 and four CPu areas (dorsal, ventral, medial and lateral; cf. e.g. [6, 14, 21]; see Figure 1).

142 These images were taken bilaterally from the two consecutive sections, resulting in four

143 samples per region per mouse. Stained nuclei were identified by dark nuclear coloration

144 surrounded by lighter stained cytoplasm (Figure 2), published images of successful

$145 \mathrm{FosB} / \Delta$ FosB immunolabeling being used as guides [23]. Dead cells were also counted

146 (because these could act as confounds by preventing positive staining), easily identified

147 via nuclear condensation (see Figure 2). Both were counted manually.

148 Because of weak staining compared to published studies [e.g. 14, 23] and

149 concerns over cell deaths, we first ran extensive checks for data quality and consistency

150 of staining. This included assessing the scorer (DP)'s internal consistency, a subset of 25

151 regions being randomly selected for a blind re-count of cells positively stained for

$152 \mathrm{FosB} / \Delta \mathrm{Fos} B$ and dead cells. Here, as throughout, data were analysed using General

153 Linear Models (GLMs) in JMP 12.0, and Box-Cox transformed when needed to meet the

154 assumptions of parametric statistics (Spearman's tests being used when this was

155 unsuccessful). First counts strongly predicted blind re-counts $(\mathrm{P}<0.0001$ for both cell

156 types) indicating high intra-rater reliability. Next, relationships between positively stained

157 and dead cell counts between the two consecutive sections per hemisphere were assessed.

158 Both positive counts and dead cells counts from successive sections positively correlated

159 ( $\mathrm{P}<0.05$ in all tests), save for the right hemisphere's NAc ( $\mathrm{P}>0.05$ in both tests).

160 Because successive sections were thus generally similar, values from each pair were

161 averaged for subsequent analyses. A series of GLMs then assessed whether positive

162 counts for each region covaried between the two hemispheres. Dead cell counts were

163 statistically controlled for by being added as covariates (since they compromised staining,

164 samples with high dead counts having low positive counts: $\mathrm{P}<0.05$ in all analyses; also

165 see Fig. 4a). Positive counts significantly covaried between hemispheres for the NAc, and

166 dorsal and lateral CPu regions, and showed a strong similar trend in the medial $\mathrm{CPu}(\mathrm{P}<$

1670.054 in all tests). Because overall, staining levels thus typically covaried between

168 hemispheres, right and left hemisphere values were averaged for subsequent analyses.

169 Finally, inter-relationships between positive counts in all four CPu sub-regions were

170 investigated for staining consistency, again statistically controlling for dead cells. All 
171 significantly covaried ( $\mathrm{P}<0.05$ in all tests), and so were averaged for subsequent 172 analyses. By contrast, none covaried with NAc counts ( $\mathrm{P}>0.05$ in all analyses).

173 Investigating the relationships between $\mathrm{SB}$ and basal ganglial FosB/ $\Delta \mathrm{FosB}$

174 staining revealed no significant relationship between SB and positively stained cell

175 counts within the $\mathrm{CPu}\left(\mathrm{F}_{1,8}=0.53, \mathrm{p}=0.49\right)$. To explore further, SB was regressed against

176 the positive counts for each individual CPu region, but again, no relationships were found

177 ( $\mathrm{P}>0.05$ in all tests). However, there was a positive correlation with SB in the NAc $\left(\mathrm{F}_{1,8}\right.$

$178=9.27, \mathrm{p}=0.016)$. This GLM again statistically controlled for dead cell counts, and also

179 used Box-Cox transformed data, making this significant relationship hard to convey in a

180 figure. Raw data were therefore plotted and analysed with a Spearman's test (see Figure

181 3). The analysis was also re-run in a two-step manner conceptually similar to the GLM

182 but, again, easier to present graphically (see Figures 4a and 4b).

183 Our findings thus implicate the ventral striatum in the cage-induced SB of C57

184 laboratory mice, consistent with Cabib’s hypothesis [3, 10] and suggesting an aetiology

185 similar to that of 'hyper-motivated' compulsive gambling, drug-taking and eating, and

186 stimulant-induced hyper-activity [1, 2, 5, 25]. Our primary interest was to identify

187 structures playing a causal role in SB. Elevated striatal (especially NAc) $\Delta$ FosB does

188 influence behaviour, for instance promoting reward-seeking, wheel-running and

189 impulsivity in food-rewarded tasks (e.g. [16, 25]). We may therefore have successfully

190 identified a cause of SB. However, we cannot assume this type of causality from mere

191 correlation. Indeed, caution is urged by the way that NAc FosB elevates in response to

192 motivationally salient stimuli, both stressors and rewards [14-16, 25]): findings that

193 suggest two alternative explanations for our results. One is that NAc $\Delta$ FosB and SB are

194 independently increased by stress, with no causal connection between them; thus high SB

195 mice find their cages particularly stressful (which elevates $\Delta \mathrm{FosB}$ ), and are highly

196 motivated to escape (escape-attempts developing into SB: [4]). Alternatively, performing

197 SB could be rewarding, such that its performance causes increased NAc FosB. Careful

198 experimental work is now needed to distinguish these hypotheses.

199 Our findings further suggest that the aetiology of this SB diverges from classic

200 amphetamine stereotypies or other repetitive behaviours induced by motor loop over-

201 activation [1, 5, 7]. Completely discounting involvement of the dorsal striatum is 
202 premature given our small sample size, but, if present, its effects are clearly relatively

203 weak. This conflicts with suggestions that C57 SB reflects motor loop dysfunction [5].

204 One possible explanation is that response repetition in gambling tasks [5] is simply not a

205 good diagnostic of this, instead being sensitive to NAc activity. However, it is also

206 possible that the two sub-populations of C57s studied differed in the neurological bases

207 of their SB. For example, the Garner work [5] used mice that, at 5-15 months of age,

208 were largely older than ours; furthermore both our studies pooled several types of SB

209 (e.g. jumping, route-tracing and bar-mouthing) that could have occurred in differing

210 ratios: important if these diverse forms have heterogeneous aetiologies. In the future we

211 therefore plan to replicate this promising use of FosB/ $\Delta$ FosB immunohistochemistry to

212 understand SB, combining it with finer distinctions between different SB forms, along

213 with measures of both response repetition in gambling tasks (cf. [5]) and impulsive

214 responses to rewards (cf. [1, 10, 25]).

215

216 Acknowledgments

217 Many thanks to the NSERC Discovery programme for funding GJM \& EC; the Ontario

218 Graduate Scholarship programme for supporting LH; and the Fyssen Fellowship scheme

219 for supporting CF. Very useful technical advice was provided by Craig Bailey

220 (University of Guelph), Paolo Campus (University of Rome) and Pietro Paletta

221 (University of Guelph). Maka Natsvlishvili provided expert care of the mice. 


\section{Table 1: Behaviour recorded during scan-sampling}

\begin{tabular}{|c|c|}
\hline Category & Description \\
\hline \multirow[t]{3}{*}{$\begin{array}{l}\text { Stereotypic } \\
\text { behaviour }\end{array}$} & $\begin{array}{l}\text { Bar mouthing: mouse holds cage bar in mouth for } 1 \text { s or longer while } \\
\text { making movements along it }\end{array}$ \\
\hline & $\begin{array}{l}\text { Route tracing: mouse runs over cage floor in a pattern for three or more } \\
\text { repetitions }\end{array}$ \\
\hline & $\begin{array}{l}\text { Patterned climbing: climbing on cage lid in a pattern for three or more } \\
\text { repetitions }\end{array}$ \\
\hline Other Activity & $\begin{array}{l}\text { Moving, digging, carrying/manipulating nest materials; moving across cage } \\
\text { in a non-stereotypic way; grooming; movement in nest/shelter although out } \\
\text { of clear sight of experimenter; feeding; drinking; chasing or being chased; } \\
\text { displacing another mouse from the feeder, or being displaced. }\end{array}$ \\
\hline
\end{tabular}

Inactive

Mouse is still (this includes eyes closed presumed sleeping; eyes open; and out of sight but not moving) 
232 Figure 1: Diagram of coronal section of mouse brain (at bregma +1.10 , modified from

233 [14]), showing where dorsal CPu (1), medial CPu (2), ventral CPu (3), lateral CPu (4) and

234 NAc (5) images were sampled (the latter not aiming to distinguish core from shell, but

235 instead to approximately span both regions). 'lv' = lateral ventricle; 'ac' = anterior

236 commissure.

238 Figure 2: One typical image (see text for details), here of medial caudate-putamen, 239 highlighting four examples of $\mathrm{Fos} B / \Delta$ FosB stained nuclei (filled arrows) and two

240 examples of dead cells (unfilled arrows). NEEDS SCALE

Figure 3: Counts of cells in the NAc that positively stained for FosB/ $\Delta$ FosB, plotted against SB level (Spearman rho $=0.67, \mathrm{P}<0.05$ ). For how this positive relationship improves if dead cell counts are statistically controlled for, please see text and Figures 4a 246 and $b$.

Figure 4a: Counts of cells that were positively stained for $\operatorname{FosB} / \Delta \mathrm{Fos} B$, or dead, in the NAc of each mouse, along with the line of best fit (dashed) for this negative relationship. The values by each datapoint show that mouse’s SB level. The "residual” measure for positive counts is the vertical distance between each datapoint and the line (e.g. as shown by the vertical dotted line for the subject with 18.5\% SB). Positive residuals thus mean

253 high positive counts of FosB/ $\Delta$ FosB stained cells for the number of dead cells in any 254 given sample, while negative residuals mean low positive counts for the number of dead 255 cells. By inspection, the more stereotypic mice have more positive residuals (tested 256 statistically in Fig. 4b).

258 Figure 4b: Residual values for positive counts of FosB/ $\Delta$ FosB stained cells in the NAc 259 (positive residuals - those to the right of the dashed line - meaning many positive counts 
260 for the number of dead cells in a given sample), plotted against each animal's SB. The 261 asterisk indicates the 18.5\% SB mouse highlighted in Fig. 4a. Mice with higher residual 262 values are more stereotypic (Spearman rho $=0.77, \mathrm{P}<0.01$ ): an effect stronger than that 263 in Fig. 3 because of this correction for dead cell counts (cf. the GLM reported in the text). 


\section{References}

267 1). McBride, S. D., \& Parker, M. O. (2015). The disrupted basal ganglia and behavioural

268 control: An integrative cross-domain perspective of spontaneous stereotypy. Behav. Brain 269 Res. 276: 45-58.

270 2). Muehlmann, A. M., \& Lewis, M. H. (2012). Abnormal repetitive behaviours: shared

271 phenomenology and pathophysiology. J. Intellect. Disabil. Res. 56: 427-440.

272 3). Cabib S. \& N. Bonaventura (1997). Parallel strain-dependent susceptibility to

273 environmentally-induced stereotypies and stress-induced behavioral sensitization in mice.

274 Physiol. Behav. 61: 499-506, 1997

275 4). Campbell, D. L., Dallaire, J. A \& Mason, G. J. (2013). Environmental enrichment

276 reduces perseveration in the American mink, but enhances spontaneous alternation.

277 Behav. Brain Res. 239: 177-187.

278 5). Garner, J. P., Thogerson, C. M., Dufour, B. D., et al. (2011). Reverse-translational

279 biomarker validation of Abnormal Repetitive Behaviors in mice: An illustration of the

280 4Ps modeling approach. Behav. Brain Res. 219: 189-196

281 6). Tanimura, Y., Vaziri, S., \& Lewis, M. H. (2010). Indirect basal ganglia pathway

282 mediation of repetitive behavior: attenuation by adenosine receptor agonists. Behav.

283 Brain Res. 210: 116-122.

284 7). Lewis, M. H., et al. (2007). Animal models of restricted repetitive behavior in autism.

285 Behav. Brain Res. 176: 66-74.

286 8). Güldenpfennig, M., De Wet, W., du Preez, J. et al. (2011). Cortico-striatal oxidative

287 status, dopamine turnover and relation with stereotypy in the deer mouse. Physiol. Behav. 288 103: 404-411.

289 9). Garner, J. P. (2005). Stereotypies and other abnormal repetitive behaviors: potential 290 impact on validity, reliability, and replicability of scientific outcomes. ILAR J. 46: 106291117.

292 10). Cabib, S. (2006). Neurobiology of stereotypy II: The role of stress. In: G. Mason \&

293 J. Rushen (eds.) Stereotypic Behaviour in Captive Animals: Fundamentals and

294 Applications to Welfare (second edition). CAB International, Wallingford, pp. 227-254. 
11). Moy, S. S., Riddick, N. V., Nikolova, V. D., et al. (2014). Repetitive behavior profile and supersensitivity to amphetamine in the C58/J mouse model of autism. Behav. Brain Res. 259: 200-214.

298 12). Latham, N., \& Mason, G. (2010). Frustration and perseveration in stereotypic captive 299 animals: Is a taste of enrichment worse than none at all? Behav. Brain Res 211: 96-104.

300 13). Gross, A. N., Engel, A. K. J., Richter, S. H., et al. (2011). Cage-induced stereotypies in female ICR CD-1 mice do not correlate with recurrent perseveration. Behav. Brain

302 Res. 216: 613-620.

303 14). Frenois, F., Morreao, M., O’Connor, J., et al. (2007). Lipopolysaccharide induces

304 delayed FosB/DeltaFosB immunostaining within the mouse extended amygdala,

305 hippocampus and hypothalamus, that parallel the expression of depressive-like behaviour.

306 Psychoneuroendocrinol. 32: 516-531.

307 15). Nishijima, T., Kawakami, M., \& Kita, I. (2012). Long-term exercise is a potent

308 trigger for $\triangle$ FosB induction in the hippocampus along the dorso-ventral axis. PloS

309 one, 8(11), e81245-e81245.

310 16). Lobo, M. K., Zaman, S., Damez-Werno, D. M., et al. (2013). $\Delta$ FosB induction in 311 striatal medium spiny neuron subtypes in response to chronic pharmacological, 312 emotional, and optogenetic stimuli. Journal of Neuroscience 33: 18381-18395.

313 17). Walker, M., Fureix, C., Palme, R. et al. (2013). Co-housing rodents with different 314 coat colours as a simple, non-invasive means of individual identification: Validating 315 mixed-strain housing for C57BL/6 and DBA/2 mice. PLOS One 8: e77541.

316 18). Harper, L., Choleris, E. Ervin, K. et al. (2015). Stereotypic mice are aggressed by

317 their cagemates, and tend to be poor demonstrators in social learning tasks. Animal

318 Welfare 24: 463-473.

319 19). Campbell, P., Reep, R., Stoll, M., et al. (2009). Conservation and diversity of Fosxp2 320 expression in muroid rodents: Functional implications. J. Comp. Neurol. 512: 84-100.

321 20). Lazic, S. E. (2010). The problem of pseudoreplication in neuroscientific studies: is it 322 affecting your analysis? BMC Neuroscience, 11: 5.

323 21). Olsen, Y. Huang, S. Goodwin, D. et al. (2008). Microarray analysis reveals 324 distinctive signaling between the bed of the nucleus of the stria terminalis, nucleus 325 accumbens and dorsal striatum Physiol. Genom. 32: 283-298. 
326 22). Kaplan, G. B., Leite-Morris, K. A., Fan, W., et al. (2011). Opiate sensitization

327 induces FosB/DeltaFosB expression in prefrontal cortical, striatal and amygdala brain

328 regions. PloS One, 6: e23574.

329 23). Conversi, D., Bonito-Olivia, A., Orsini, C. \& Cabib, S. (2006). Habituation to the

330 test cage influences amphetamine-induced locomotion and Fos expression and increases

331 FosB/AFosB-like immunoreactivity in mice. Neuroscience 141: 597-605.

332 24). Ozburn, A., Mayfield, R., Ponomarev, I., et al. (2012). Chronic self-administration

333 of alcohol results in elevated $\triangle$ FosB: Comparison of hybrid mice with distinct drinking

334 patterns. BMC Neuroscience 13(130): doi:10.1186/1471-2202-13-130

335 25). Velázquez-Sánchez, C., Ferragud, A., Moore, C. F., et al. (2014). High trait

336 impulsivity predicts food addiction-like behavior in the

337 rat. Neuropsychopharmacology 39: 2463-2472. 
$1.10 \mathrm{~mm}$

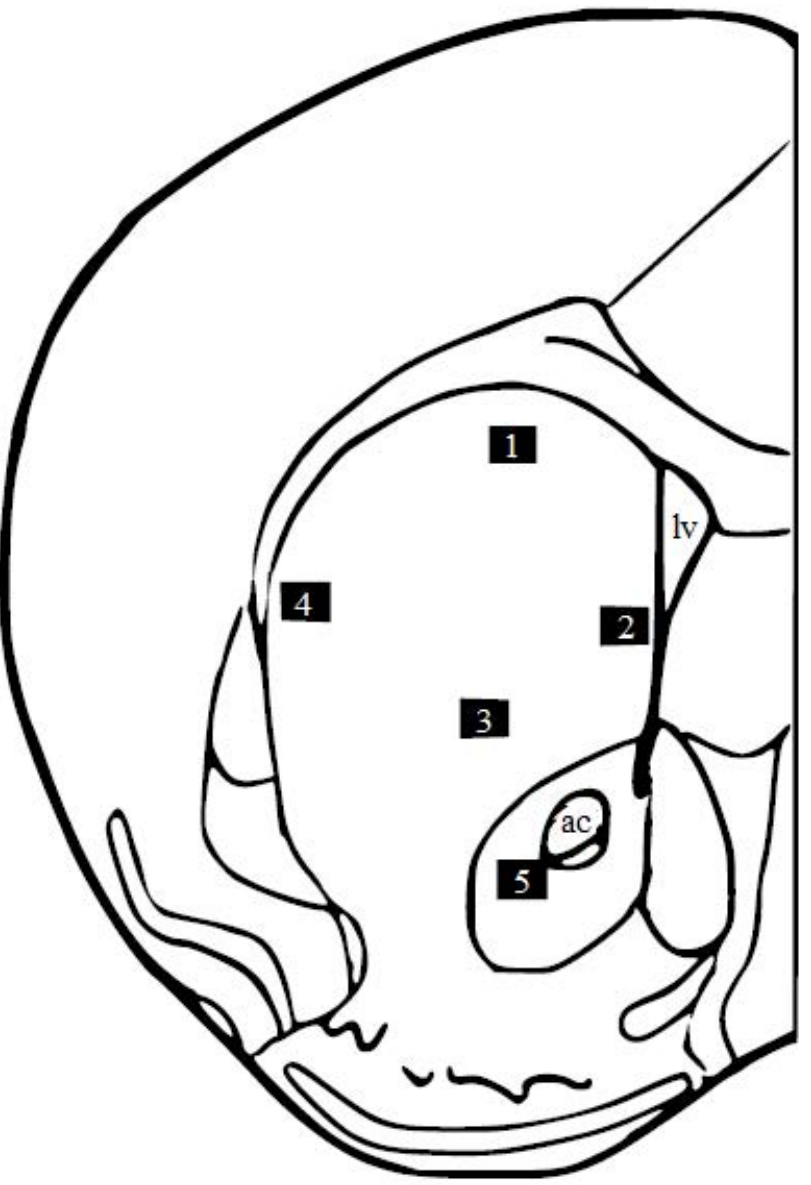




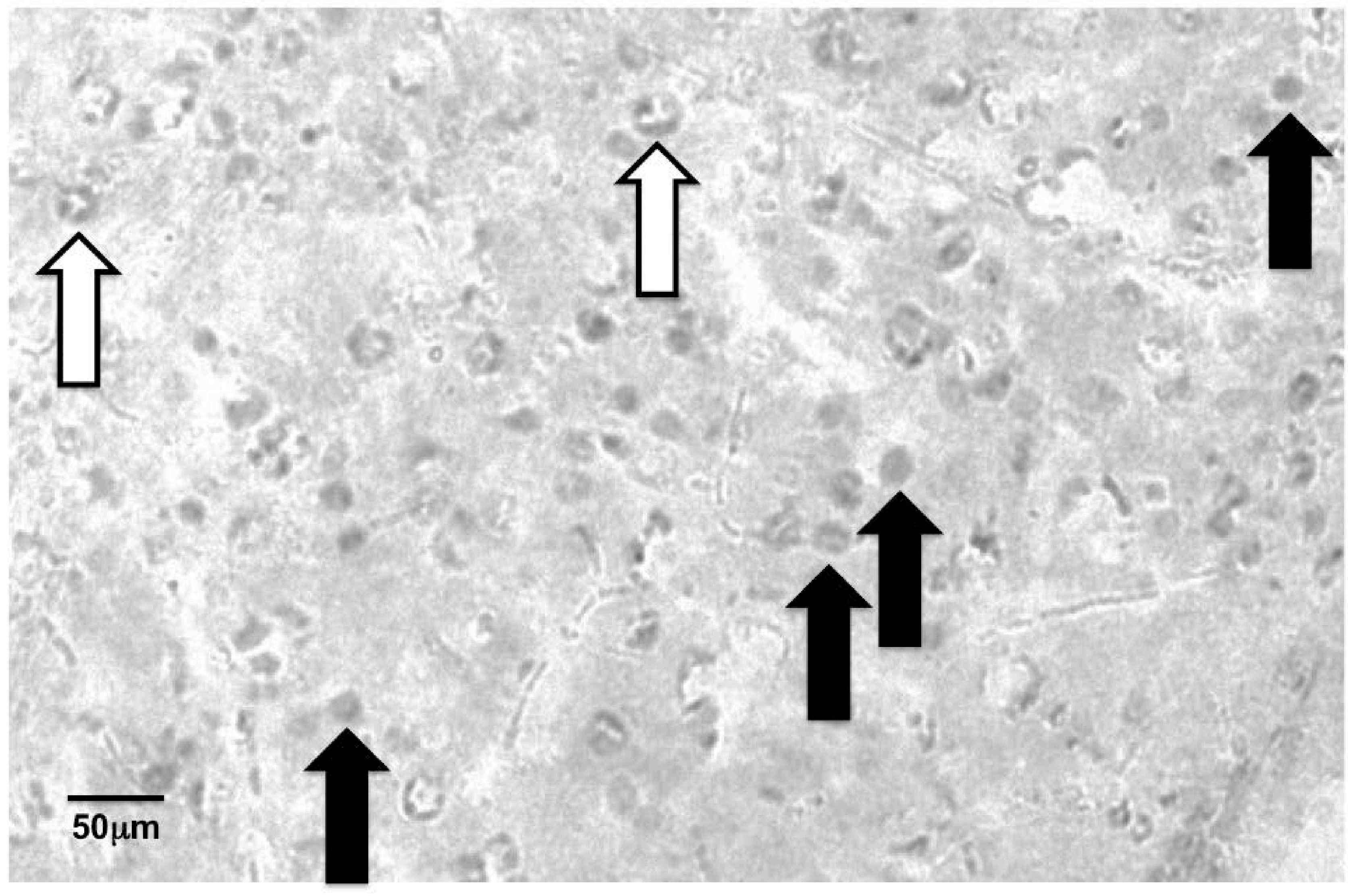




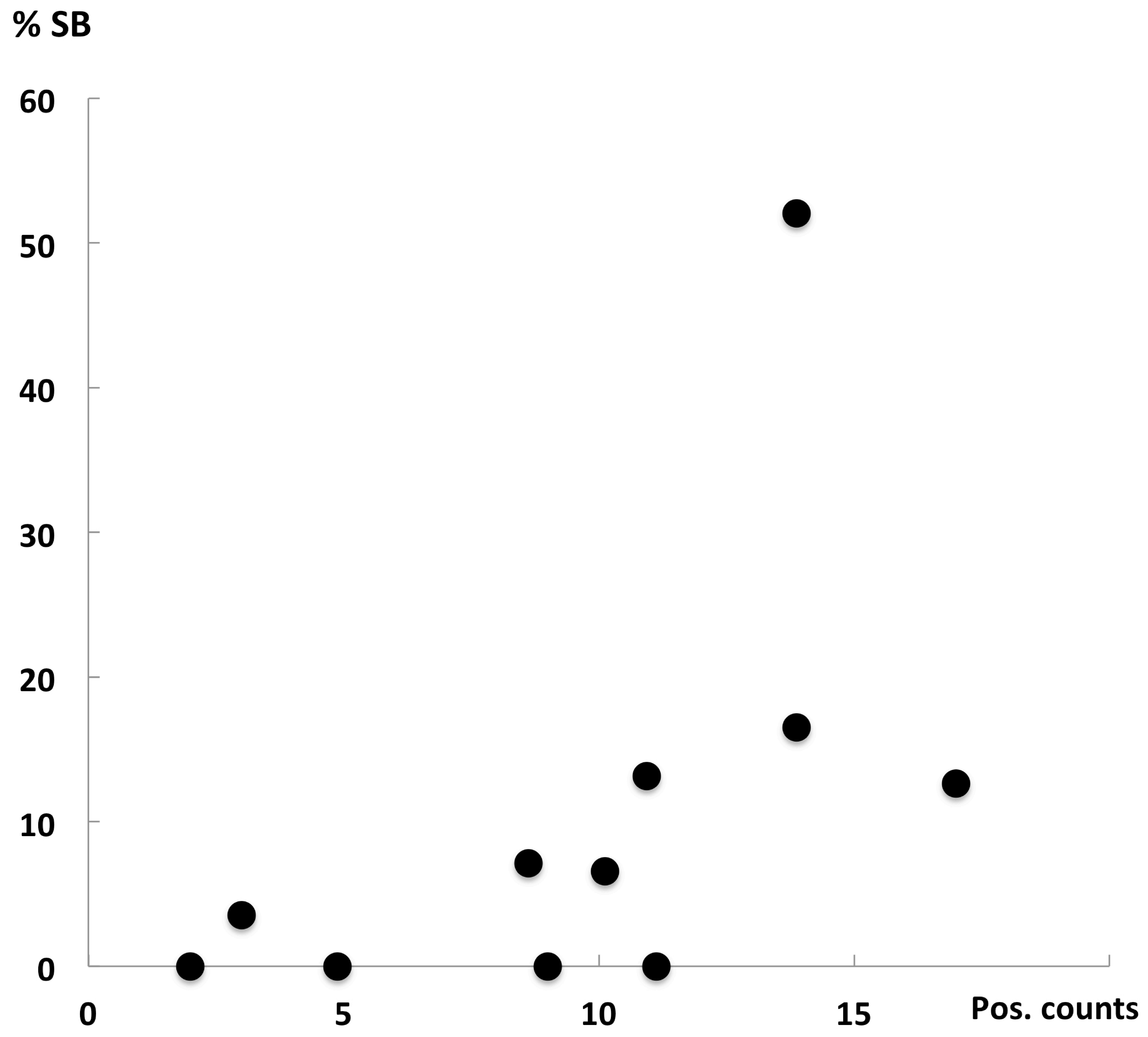


Pos.

counts

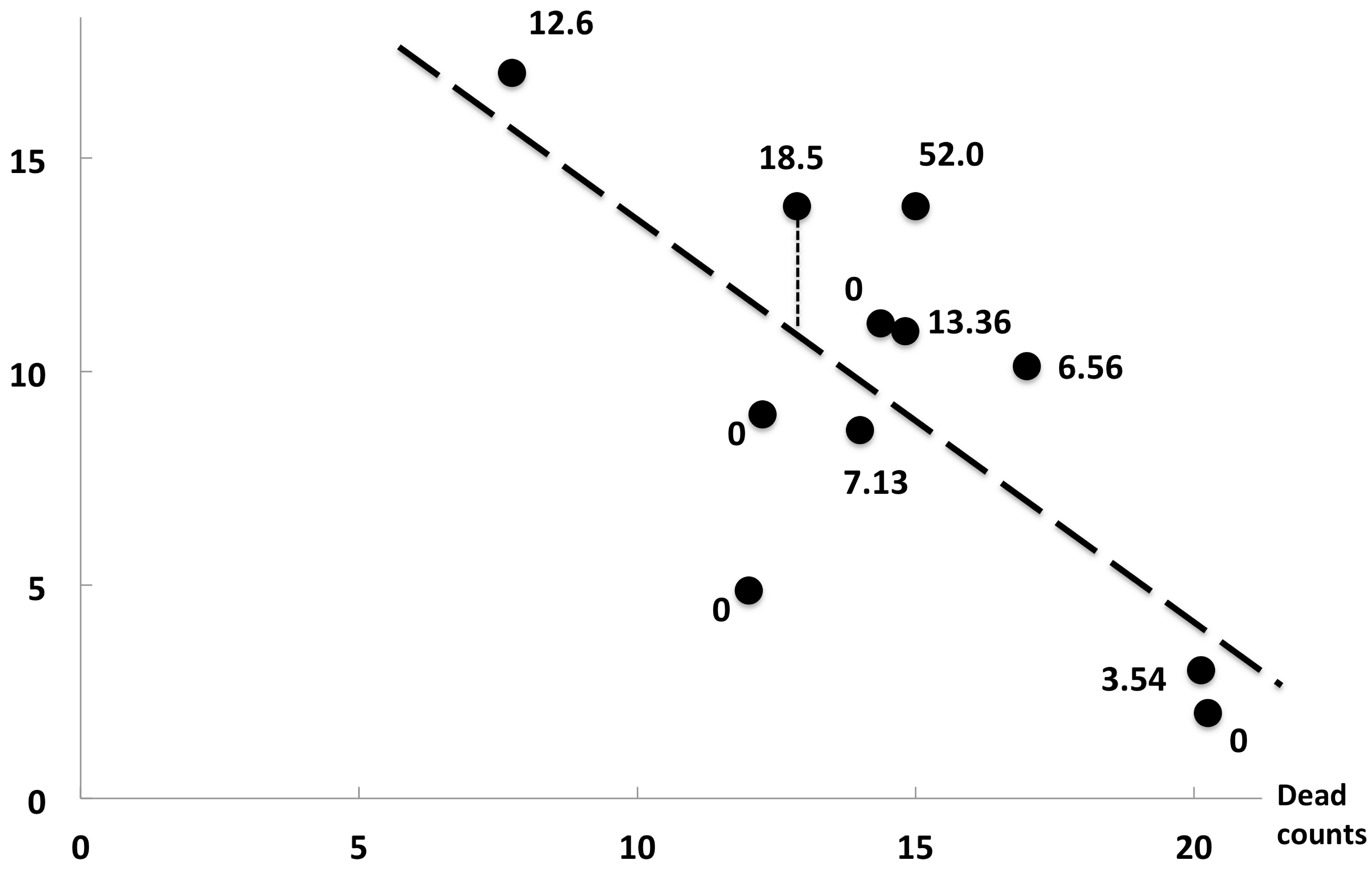




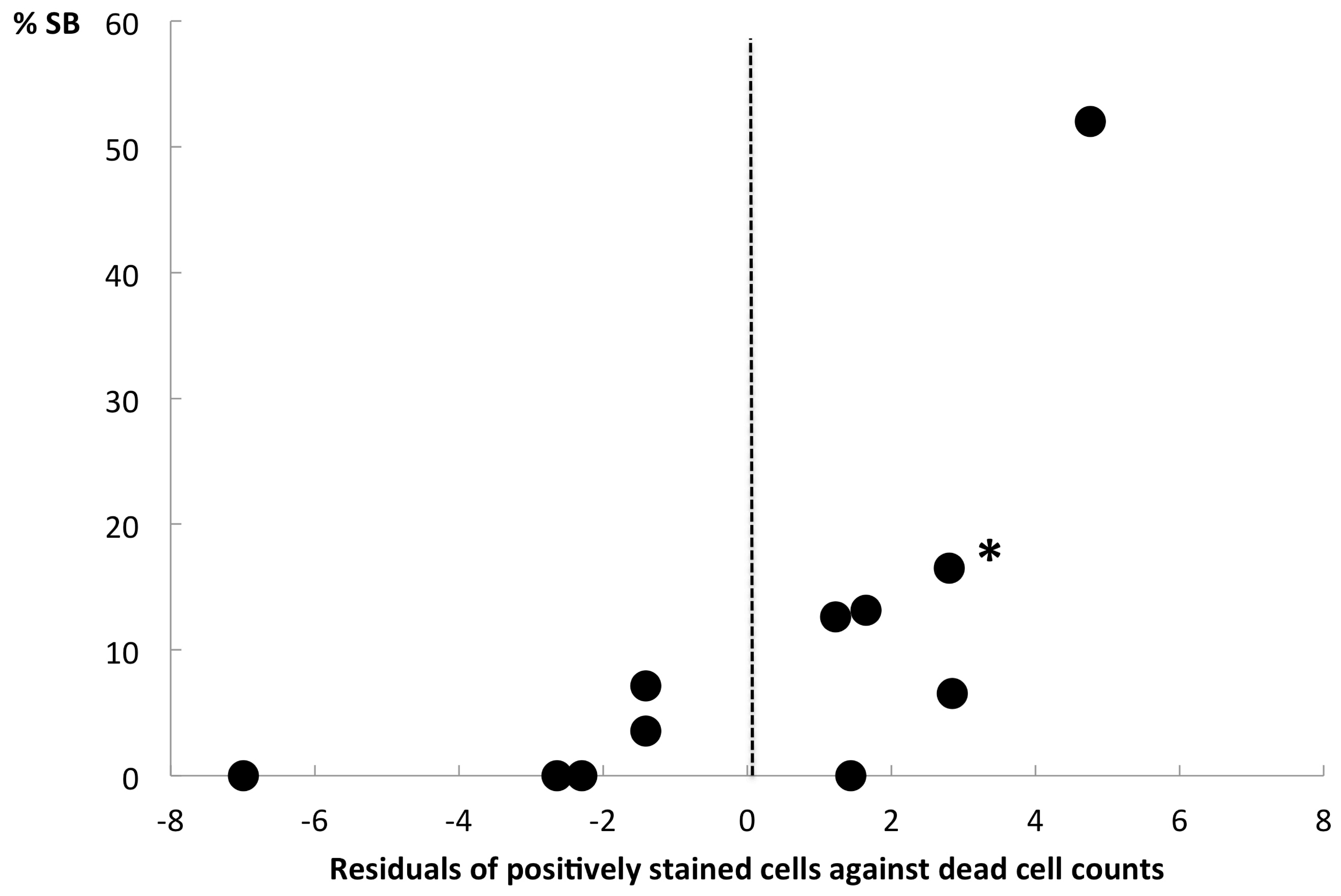

